\title{
Quadrupole Excitation of Ions in Linear Quadrupole Ion Traps with Added Octopole Fields
}

\author{
XianZhen Zhao, Ori Granot, and D. J. Douglas \\ Department of Chemistry, University of British Columbia, Vancouver, British Columbia, Canada
}

Modeling and experimental studies of quadrupole excitation of ions in linear quadrupole traps with added octopole fields are described. An approximate solution to the equations of motion of ions trapped in a quadrupole with added octopole and dodecapole fields, with quadrupole excitation and damping is given. The solutions give the steady-state or stationary amplitudes of oscillation with different excitation frequencies. Trajectory calculations of the oscillation amplitudes are also presented. The calculations show that there can be large changes in the amplitude of ion oscillation with small changes in excitation frequency, on both the low and high-frequency sides of a resonance. Results of experiments with quadrupole excitation of reserpine ions in linear quadrupole traps with $2.0 \%, 2.6 \%$, and $4.0 \%$ added octopole fields are given. It is found that as the excitation frequency is changed, two resonances are generally observed, which are attributed to the motion in the $\mathrm{x}$ and $\mathrm{y}$ directions. The two resonances can have quite different intensities. Sudden jumps or sharp sided resonances are not observed, although in some cases asymmetric resonances are seen. The calculated frequency differences between the two resonances are in approximate agreement with the experiments. (J Am Soc Mass Spectrom 2008, 19, 510-519) (0 2008 American Society for Mass Spectrometry

L inear quadrupole ion traps are finding widespread applications in mass spectrometry [1]. For tandem mass spectrometry, where ions are excited at their resonant frequencies in the presence of a collision gas to induce fragmentation, the addition of field distortions, described by the addition of higher multipoles to the potential, can increase the fragmentation efficiency, particularly at low pressures $[2,3]$. The spatial part of the potential in a linear quadrupole with field distortions can be written as a superposition of multipoles

$$
\Phi\left(x_{r}, y_{r}\right)=\operatorname{Re}\left[\sum_{N=0}^{\infty} A_{N}\left(\frac{x_{r}+i y_{r}}{r_{0}}\right)^{N}\right]
$$

where $\operatorname{Re}[f u n c]$ is the real part of the complex function func and $A_{N}$ is the dimensionless amplitude of a $2 \mathrm{~N}$ pole field. If no dc voltages are applied between the rods, the time-dependent potential is

$$
V\left(x_{r}, y_{r}, t_{r}\right)=\Phi\left(x_{r}, y_{r}\right) V_{r f} \cos \left(\Omega t_{r}\right)
$$

where $V_{r f}$ is the zero to peak rf voltage pole to ground, $r_{0}$ the field radius, $x_{r}, y_{r}$, are Cartesian coordinates, and $\mathrm{t}_{r}$ time. The addition of higher multipoles can lead to nonlinear dynamics, giving sudden changes in the

Address reprint requests to Dr. D. J. Douglas, Department of Chemistry, University of British Columbia, 2036 Main Mall, Vancouver, BC, V6T 1Z1, Canada. E-mail: douglas@chem.ubc.ca amplitude of ion oscillation with small changes in excitation frequency. These effects have been described in detail for dipole excitation of ions in a linear quadrupole trap with a $4 \%$ added octopole field [2a].

Trapped ions can also be excited by quadrupole excitation, sometimes called parametric excitation [4]. With quadrupole excitation, the excitation electric field strength is proportional to the distance off axis. In a pure quadrupole field, ions are excited when the excitation angular frequency $\omega_{e x}$ is

$$
\omega_{e x}=|n+\beta| \frac{\Omega}{K}
$$

where $n=0, \pm 1, \pm 2 \ldots, K=1,2,3, \ldots, \Omega$ is the angular frequency of the trapping radiofrequency field, and $\beta$ is a function of the Mathieu parameter $q$ given by

$$
q=\frac{4 z e V_{r f}}{m \Omega^{2} r_{0}^{2}}
$$

with $e$ the charge of an electron, and $z$ the number of charges on an ion. The effects of nonlinear dynamics can also be seen with quadrupole excitation. Werth and coworkers have reported large changes in oscillation amplitude and strongly asymmetric resonances with parametric excitation of ions in 3D Paul traps [5-7] and electrons in Penning traps [8,9]. These effects were attributed to the higher order fields of the traps. 
Table 1. Rod dimensions and calculated harmonic amplitudes, $R_{x}=r_{0}$. The rod sets used in the experiments here have $r_{0}=4.5$ mm

\begin{tabular}{|c|c|c|c|c|c|c|c|}
\hline Nominal \% octopole & $R_{y} / R_{x}$ & $A_{0}$ & $A_{2}$ & $A_{4}$ & $A_{6}$ & $A_{8}$ & $A_{10}$ \\
\hline 2.0 & 1.220 & -0.02029 & 0.9994 & 0.01961 & 0.003281 & 0.0008604 & -0.002345 \\
\hline 2.6 & 1.300 & -0.02638 & 1.0014 & 0.02567 & 0.001286 & 0.0009514 & -0.002358 \\
\hline 4.0 & 1.516 & -0.0403 & 1.0056 & 0.03980 & -0.002990 & 0.0008702 & -0.002319 \\
\hline
\end{tabular}

Here we describe modeling and experimental studies of quadrupole excitation of ions in linear quadrupole traps with added octopole fields. We give an approximate solution to the equations of motion of ions trapped in a quadrupole with added octopole and dodecapole fields with quadrupole excitation and damping. The solutions give the steady-state or stationary amplitudes of oscillation with different excitation frequencies. Trajectory calculations of the oscillation amplitudes are also presented. The results show that there can be large changes in the amplitude of ion oscillation with small changes in excitation frequency. These large changes appear on both the low and highfrequency sides of a resonance, and thus might be used to produce high-resolution in isolating ions [2a, 10], the main motivation for this work. We then describe an experimental investigation of quadrupole excitation of ions in linear quadrupole traps with $2.0 \%, 2.6 \%$, and $4.0 \%$ added octopole fields. Protonated ions of reserpine are trapped at pressures of 1.0 and 0.2 mTorr of nitrogen and excited at the resonances with $K=1$ and 2. Our principal finding is that as the excitation frequency is scanned, two resonances are generally observed, which we attribute to the motion in the $x$ and $y$ directions. The two resonances can have quite different intensities. Sudden jumps or sharp sided resonances are not observed, although in some cases asymmetric resonances are seen. The calculated frequency differences between the two resonances are in approximate agreement with the experiments.

\section{Theoretical Treatment}

An octopole field can be added to a round rod quadrupole by making the radius of the y rods, $R_{y}$, greater than the radius of the $x$ rods, $R_{x}$ [11]. All rods are equally spaced a distance $r_{0}$ from the quadrupole axis. The ratio $R_{y} / R_{x}$ determines the magnitude of the added octopole field. Smaller amounts of higher even multipoles are also added. The ratios $R_{y} / R_{x}$ and multipole amplitudes, $A_{N}$, of the rod sets modeled and used in the experiments here are shown in Table 1 . Note the rod sets have an axis potential $A_{0} V_{r f}$ where $A_{0} \approx-A_{4}$.

The equations of motion of an ion in the potential of eq 2 cannot be solved analytically. To gain some insight into the ion motion, we use a perturbation method to calculate the stationary oscillation amplitudes of an ion in the pseudo- or effective potential arising from the time-dependent potential of eq 2 with quadrupole excitation and damping.
The effective potential, $V_{e f f}\left(x_{r}, y_{r}\right)$ is determined by the electric fields arising from the potential of eq 2 and is given by [12],

$$
\begin{aligned}
V_{e f f}\left(x_{r}, y_{r}\right)= & \frac{z e \vec{E}^{2}}{4 m \Omega^{2}} \\
= & \frac{z e}{4 m \Omega^{2}}\left[\left(\frac{\partial \Phi\left(x_{r}, y_{r}\right)}{\partial x_{r}}\right)^{2}+\left(\frac{\partial \Phi\left(x_{r}, y_{r}\right)}{\partial y_{r}}\right)^{2}\right] \\
= & \frac{z e V_{r f}^{2}}{4 m \Omega^{2} r_{0}^{2}}\left[4 A_{2}^{2} \frac{x_{r}^{2}+y_{r}^{2}}{r_{0}^{2}}+16 A_{2} A_{4} \frac{x_{r}^{4}-y_{r}^{4}}{r_{0}^{4}}\right. \\
& \left.+\left(16 A_{4}^{2}+24 A_{2} A_{6}\right) \frac{x_{r}^{6}+y_{r}^{6}}{r_{0}^{6}}+\ldots\right]
\end{aligned}
$$

The equation of motion of an ion in this effective potential with quadrupole excitation and collisional damping is

$$
m \frac{d^{2} \vec{r}}{d t_{r}^{2}}=-z e \nabla V_{e f f}-z e \nabla \Phi_{e x}-\Gamma \frac{d \vec{r}}{d t_{r}}
$$

where $\vec{r}=x_{r} \hat{\imath}+y_{r} \hat{\jmath}$ is the position vector, and $\hat{\imath}$ and $\hat{\jmath}$ are unit vectors in the $\mathrm{x}$ and $\mathrm{y}$ directions. The quadrupole excitation potential $\Phi_{e x}$ is

$$
\Phi_{e x}\left(x_{r}, y_{r}, t_{r}\right)=\Phi\left(x_{r}, y_{r}\right) V_{e x} \cos \left(\omega_{e x} t_{r}\right)
$$

where $\omega_{e x}=2 \pi f_{e x}$ is the angular frequency of the quadrupole excitation. The damping coefficient $\Gamma$ due to ion collisions with the background gas is [2a].

$$
\Gamma=\frac{3.01 \sigma n m_{2} \sqrt{2 k_{B} T / m_{2}}}{2}=\frac{3.01 P \sigma \sqrt{2 m_{2} /\left(k_{B} T\right)}}{2}
$$

where $n$ is the background gas number density, $m_{2}$ is the mass of the background gas, $P$ is the background gas pressure, $\sigma$ is the collision cross section, $k_{B}$ is Boltzmann's constant, and $T$ is the gas temperature.

In general, the ion motions in the $\mathrm{x}$ and $\mathrm{y}$ directions are coupled by the higher multipole fields. However, motions along the $\mathrm{x}$ axis (i.e., $x_{r}$ when $y_{r}=0$ ) and along the y axis $\left(y_{r}\right.$ when $\left.x_{r}=0\right)$ are uncoupled. Including the nonlinear terms up to $x^{5}$ and $y^{5}$ but retaining only the quadrupole excitation terms, the equations of motion are

$$
\frac{d^{2} x}{d t^{2}}+\lambda \frac{d x}{d t}+[1-\gamma \cos (\omega t)] x+B_{3} x^{3}+B_{5} x^{5}=0
$$




$$
\frac{d^{2} y}{d t^{2}}+\lambda \frac{d y}{d t}+[1+\gamma \cos (\omega t)] y-B_{3} y^{3}+B_{5} y^{5}=0
$$

Here we have introduced the dimensionless variables $x$, $y, t, \omega, \lambda, \gamma, B_{3}$, and $B_{5}$.

The dimensionless coordinates, time, and excitation frequency are defined as

$$
x=\frac{x_{r}}{r_{0}} ; y=\frac{y_{r}}{r_{0}} ; t=\omega_{0} t_{r} ; \omega=\frac{\omega_{e x}}{\omega_{0}}
$$

where

$$
\omega_{0}=2 \pi f_{0}=\frac{q A_{2}}{\sqrt{8}} \Omega
$$

is the secular frequency of ion oscillation in a quadrupole potential. With these definitions, the calculated angular frequencies and angular frequency shifts are in units of the secular frequency $\omega_{0}$, and the positions of ions and the ion oscillation amplitudes are in units of the field radius $r_{0}$. The dimensionless damping and driving coefficients, $\lambda$ and $\gamma$ are defined as

$$
\begin{aligned}
& \lambda=\frac{\Gamma}{m \omega_{0}}=\frac{3.01 P \sigma \sqrt{2 m_{2} /\left(k_{B} T\right)}}{m \omega_{0}} \\
& \gamma=\frac{2 z e V_{e x}}{m \omega_{0}^{2} r_{0}^{2}}
\end{aligned}
$$

The dimensionless constants $B_{3}$ and $B_{5}$ are determined by the multipole components of the field. Keeping multipoles up to $N=6$,

$$
\begin{aligned}
B_{3} & =\frac{8 A_{4}}{A_{2}} \\
B_{5} & =\frac{12 A_{4}^{2}+18 A_{2} A_{6}}{A_{2}^{2}}
\end{aligned}
$$

Equations 9 and 10 are essentially the same, except for the different signs in front of $\gamma$ and $B_{3}$. Therefore, we solve eq 9, for the $\mathrm{x}$ motion, and simply state the solution for the $y$ motion.

A perturbation method is necessary to solve eq 9. To properly order the terms, we first calculate the coefficients for the conditions of the experiments described below. These are listed in Table 2. The two nonlinear terms contribute in the same (first) order of perturbation theory. Based on the magnitudes of the coefficients, eq 9 can be rewritten as

$$
\frac{d^{2} x}{d t^{2}}+\epsilon \lambda \frac{d x}{d t}+[1-\epsilon \gamma \cos (\omega t)] x+\epsilon B_{3} x^{3}+\epsilon B_{5} x^{5}=0
$$

where $\epsilon$ denotes the order of the terms and has no
Table 2. Coefficients in eq 9 calculated for an ion of $\mathrm{m} / \mathrm{z}$ 609.28 , a quadrupole with an added $4 \%$ octopole field and zero to peak excitation amplitudes of $0.30 \mathrm{~V}$ and $0.35 \mathrm{~V}$ for excitation at pressures of $0.2 \mathrm{mTorr}$ and $1 \mathrm{mTorr}$, respectively

\begin{tabular}{lcccc}
\hline Pressure & $\lambda$ & $\gamma$ & $B_{3}$ & $B_{5}$ \\
\hline \hline 0.2 mTorr & 0.00154376 & 0.0396614 & 0.318042 & -0.0354884 \\
1 mTorr & 0.00771878 & 0.0462716 & 0.318042 & -0.0354884
\end{tabular}

physical meaning. Equation 17 describes a damped and parametrically driven nearly harmonic oscillator with odd orders of nonlinear distortions to the force. The method of multiple time scales (MMS) [13] is used to find an approximate solution. In this method, independently varying time scales are assumed. The fast time scale, $T_{0}$, is the normal time scale, and the first-order slower time scale $T_{1}=\epsilon T_{0}$. Keeping only these two time scales we seek an approximate solution of the form

$$
x\left(T_{0}, T_{1}\right)=x_{0}\left(T_{0}, T_{1}\right)+\epsilon x_{1}\left(T_{0}, T_{1}\right)+o\left(\epsilon^{2}\right)
$$

where $o\left(\epsilon^{2}\right)$ denotes terms equal to or smaller than the second-order. With these two time scales, the time derivatives are

$$
\frac{d}{d t}=D_{0}+\epsilon D_{1}
$$

$$
\frac{d^{2}}{d t^{2}}=D_{0}^{2}+\epsilon 2 D_{0} D_{1}+o\left(\epsilon^{2}\right)
$$

Where $D_{0}=d d T_{0}$ and $D_{1}=d d d T_{1}$ are the derivative operators with respect to the times $T_{0}$ and $T_{1}$. Inserting eqs 18,19 , and 20 into eq 17 and equating like terms according to the orders of $\epsilon$ yields

$$
\begin{aligned}
& \epsilon^{0}: \quad D_{0}^{2} x_{0}+x_{0}=0 \\
& \epsilon^{1}: \quad D_{0}^{2} x_{1}+x_{1}=-2 D_{0} D_{1} x_{0}-\lambda D_{0} x_{0}+\gamma \cos (\omega t) x_{0} \\
& -B_{3} x_{0}^{3}-B_{5} x_{0}^{5}
\end{aligned}
$$

Equation 21 is that of a simple harmonic oscillator. We write the solution in complex form

$$
x_{0}\left(T_{0}, T_{1}\right)=A\left(T_{1}\right) e^{i T_{0}}+A^{*}\left(T_{1}\right) e^{-i T_{0}}
$$

where $A^{*}\left(T_{1}\right)$ is the complex conjugate of $A\left(T_{1}\right)$, which is a function of the slow time scale $T_{1}$. The function $A\left(T_{1}\right)$ is determined to ensure there are no secular terms in eq 22. Substituting eq 23 into the right side of eq 22 yields 


$$
\begin{aligned}
& D_{0}^{2} x_{1}+x_{1}=\left(-i \frac{d A}{d T_{1}}-i \lambda A-3 B_{3} A^{2} A^{*}\right. \\
& \left.-10 B_{5} A^{3}\left(A^{*}\right)^{2}\right) e^{i T_{0}} \\
& +\frac{\gamma}{2}\left[A e^{i(\omega+1) t}+A^{*} e^{i(\omega-1) t}\right]-B_{3} A^{3} e^{i 3 T_{0}}-B_{5}\left(A^{5} e^{i 5 T_{0}}\right. \\
& \left.+5 A^{4} A^{*} e^{i 3 T_{0}}\right)+ \text { C.C. }
\end{aligned}
$$

where C.C. stands for the complex conjugate of all the preceding terms. We consider the case where the excitation frequency is about twice that of the secular frequency ( $n=0, K=1$ in eq 3$)$. In this case, write the excitation frequency as

$$
\omega=2+\epsilon \cdot \Delta \omega
$$

where $\Delta \omega$ is the excitation frequency detuning from the primary resonant frequency, 2 (in units of $\omega_{0}$ ). Substituting eq 25 into eq 24 and removing the secular terms by equating the coefficient of term $e^{i T_{0}}$ to zero, a differential equation for $A\left(T_{1}\right)$ is obtained:

$$
\begin{aligned}
& -i 2 \frac{d A}{d T_{1}}-i \lambda A-3 B_{3} A^{2} A^{*}-10 B_{5} A^{3}\left(A^{*}\right)^{2}+\frac{\gamma}{2} A^{*} e^{i \cdot \Delta \omega \cdot T_{1}} \\
& =0
\end{aligned}
$$

Recall $A\left(T_{1}\right)$ is a complex function of $T_{1}$. Writing $A$ $\left(T_{1}\right)=a\left(T_{1}\right) e^{i \phi\left(T_{1}\right)}$ and splitting eq 26 into real and imaginary parts gives

$$
\text { Real : } \frac{d \phi}{d T_{1}}-\frac{3}{8} B_{3} a^{2}-\frac{5}{16} B_{5} a^{4}+\frac{\gamma}{4} a \cdot \cos \left(\Delta \omega \cdot T_{1}-2 \phi\right)=0
$$

$$
\text { Imaginary }:-\frac{d a}{d T_{1}}-\frac{\lambda}{2} a+\frac{\gamma}{4} a \cdot \sin \left(\Delta \omega \cdot T_{1}-2 \phi\right)=0
$$

To eliminate $T_{1}$ a variable replacement of $\Psi=$ $\Delta \omega \cdot T_{1}-2 \phi$ changes eqs 27 and 28 into

$$
\begin{aligned}
& \frac{d \Psi}{d T_{1}}=\Delta \omega-\frac{3}{4} B_{3} a^{2}-\frac{5}{8} B_{5} a^{4}+\frac{\gamma}{2} \cos \Psi \\
& \frac{d a}{d T_{1}}=-\frac{\lambda}{2} a+\frac{\gamma}{4} a \cdot \sin \Psi
\end{aligned}
$$

We now seek the stationary solutions to eqs 29 and 30 . Letting $d \psi / d T_{1}=0$ and $d a / d T_{1}=0$ and solving for $\Psi$ and $a$, one finds two possible sets of solutions. The first set of solutions is

$$
\begin{gathered}
a=0 \\
\Psi=\cos ^{-1}\left(\frac{2 \cdot \Delta \omega}{\gamma}\right)
\end{gathered}
$$

and the second set satisfies

$$
\begin{gathered}
\frac{3}{4} B_{3} a^{2}+\frac{5}{8} B_{5} a^{4}=\frac{\gamma}{2} \cos \Psi+\Delta \omega \\
\Psi=\sin ^{-1}\left(\frac{2 \lambda}{\gamma}\right)
\end{gathered}
$$

One of the most interesting things one can deduce from eq 32 is the dependence of the ion oscillation amplitudes on the excitation frequency

$$
\begin{aligned}
& \omega_{ \pm}^{x}=2 \pm \sqrt{\frac{\gamma^{2}}{4}-\lambda^{2}}+\frac{3}{4} B_{3} a_{ \pm}^{2}+\frac{5}{8} B_{5} a_{ \pm}^{4} \\
& \omega_{ \pm}^{y}=2 \pm \sqrt{\frac{\gamma^{2}}{4}-\lambda^{2}}-\frac{3}{4} B_{3} a_{ \pm}^{2}+\frac{5}{8} B_{5} a_{ \pm}^{4}
\end{aligned}
$$

The superscripts $x$ and $y$ denote the frequencies for the $\mathrm{x}$ and $\mathrm{y}$ directions, respectively. A simple comparison of eq 10 to eq 9 gives eq 34 for $\omega^{y}$. For a given set of driving and damping parameters, there are two branches labeled by the + and - subscripts, which correspond to the + and - signs in front of $\sqrt{\gamma^{2} / 4-\lambda^{2}}$, respectively. The stability of these solutions can be analyzed by considering the Jacobian matrix of eqs 29 and 30 for each of the solutions [14]. Specifically, when the eigenvalues of the matrix have a positive (negative) real part, that solution is unstable (stable).

It should also be noticed from eqs 33 and 34, in the presence of damping, there is a threshold for the excitation force to produce a resonance given by

$$
\gamma_{\min }=2 \lambda
$$

Thus the minimum excitation voltage required for resonance is

$$
\left(V_{e x}\right)_{\min }=\frac{\omega_{0} r_{0}^{2} \Gamma}{z e}
$$

The threshold for excitation of a damped harmonic oscillator with parametric excitation has been described previously [15].

Figure $1 \mathrm{a}$ and $\mathrm{b}$ show the stationary amplitudes calculated from eqs 33 and 34 for a quadrupole ion trap with a $4.0 \%$ octopole field, ions excited at $q=0.2, P=$ 1 mTorr $\left(\mathrm{N}_{2}\right)(1 \mathrm{mTorr}=0.133 \mathrm{~Pa})$, and an excitation amplitude $V_{e x}=0.40 V_{0-p}$. For Figure 1a the multipoles with amplitudes $A_{2}, A_{4}$, and $A_{6}$ (Table 1 ) are included in the calculation. Both the stable (solid lines) and unstable (dashed lines) solutions near the primary resonant 

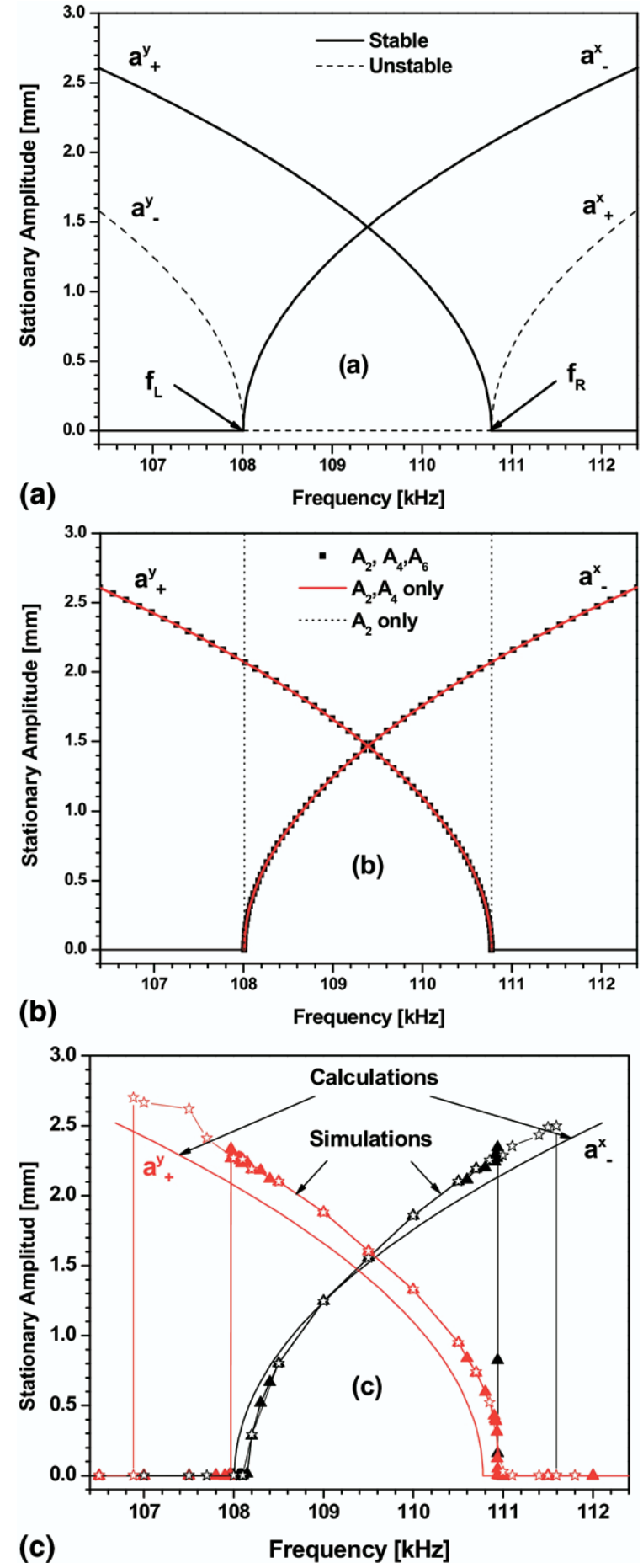

Figure 1. Oscillation amplitudes for a linear quadrupole with a $4.0 \%$ added octopole field, at a pressure $P=1 \mathrm{mTorr}^{\circ} \mathrm{N}_{2}, q=$ $0.2, V_{e x}=0.40 \mathrm{~V}_{0-p}, \Omega=2 \pi \cdot 768.107 \times 10^{3} \mathrm{~s}^{-1}, \mathrm{mz}=609.28$, gas temperature $T=295 \mathrm{~K}$, collision cross section $280 \AA^{2}$. (a) Stationary amplitude versus excitation frequency for the $\mathrm{x}$ and $\mathrm{y}$ motions with $A_{2}, A_{4}$, and $A_{6}$ included in the calculation. (b) Stationary amplitude versus excitation frequency for the $\mathrm{x}$ and $\mathrm{y}$ motions with different combinations of the multipoles included. (c) Stationary amplitudes from ion trajectories versus quadrupole excitation frequency for the $\mathrm{x}$ and $\mathrm{y}$ motions. The solid triangles are for initial positions of $x_{0}=0.1, y_{0}=0.1$; the open structure stars are for simulations with initial positions of $x_{0}=0.3, y_{0}=0.3$. frequency, for motion in the $\mathrm{x}$ (labeled $a_{ \pm}^{x}$ ) and $\mathrm{y}$ directions $\left(a_{ \pm}^{y}\right)$, are shown. For convenience two frequencies, $f_{L}$ and $f_{R}$ are defined as illustrated in Figure 1a. For frequencies $f_{e x}<f_{L}$ there are two stable branches for the y direction, $a_{+}^{y}$ and $a^{y}=0$. The solution $\mathrm{a}^{\mathrm{y}}-$ is unstable. In this region there are no resonant frequencies for the $\mathrm{x}$ motion. Conversely for frequencies $f_{e x}>$ $f_{R}$ there are two stable branches for the $\mathrm{x}$ direction, $a_{-}^{x}$ and $a^{x}=0$; in this region there are no resonant frequencies for the y motion. The solution $a_{+}^{x}$ is unstable. For frequencies $f_{L}<f<f_{k}$, both the $\mathrm{x}$ and $\mathrm{y}$ directions have stable resonant solutions, $a_{-}^{x}$ and $a_{+}^{y}$. In the regions $f_{e x}<f_{L}$ and $f_{e x}>f_{R}$ where there are two stable solutions for the $\mathrm{y}$ and $\mathrm{x}$ motions respectively, bistable behavior is possible. For a given stationary amplitude, $a_{m}$, the difference in frequency in the $\mathrm{x}$ and $\mathrm{y}$ directions for the two stable branches can be derived from eqs 33 and 34:

$$
2 \pi \cdot \Delta f=\left|2 \sqrt{\frac{\gamma^{2}}{4}-\lambda^{2}}-\frac{3}{2} B_{3} a_{m}^{2}\right|
$$

Figure $1 \mathrm{~b}$ demonstrates how the higher order multipoles influence the dependence of the stationary amplitude on the excitation frequency. Only stable solutions are shown. Between the two dotted vertical lines are the resonant frequencies for the given parameters for a pure quadrupole field $\left(A_{2}\right.$ only). There are infinitely large stationary amplitudes for these frequencies. This is characteristic of parametrically excited motion; the amplitudes increase exponentially with time at resonance. The curved thicker lines are for the case where only $A_{2}$ and $A_{4}$ are considered. The existence of the octopole term $A_{4}$ has the effect of limiting the stationary amplitudes to finite values. The amplitudes with $A_{2}, A_{4}$, and $A_{6}$ included in the calculation (the same amplitudes as in Figure 1a) are shown by the symbols. The small term $A_{6}$, (about $0.3 \%$ of the quadrupole field), makes a negligible change to the amplitudes under these conditions. The stationary amplitudes for the $\mathrm{x}$ and $\mathrm{y}$ directions are approximately symmetric about the frequency $\omega_{0}$.

\section{Trajectory Calculations}

When the ion motion with quadrupole excitation is considered in the exact potential, rather than the pseudopotential, the equation of motion can be written as

$$
m \frac{d^{2} \vec{r}}{d t_{r}^{2}}=-z e \nabla V\left(x_{r}, y_{r}, t_{r}\right)-z e \nabla \Phi_{e x}\left(x_{r}, y_{r}, t_{r}\right)-\Gamma \frac{d \vec{r}}{d t_{r}}
$$

Where $V\left(x_{r}, y_{r}, t_{r}\right)$ and $\Phi\left(x_{r}, y_{r}, t_{r}\right)$ are given by eqs 2 and 7 , respectively. Trajectory calculations were used to determine the stationary oscillation amplitudes from eq 38 . Only the quadrupole and octopole terms were included in the potential. The initial radial velocities were zero. The initial $\mathrm{x}$ and $\mathrm{y}$ positions $\left(x_{0}, y_{0}\right)$ were varied. Trajectories were calculated for 5000 cycles of the trap- 
ping rf. For each excitation frequency, after initial transients, a trajectory reaches a stationary oscillation amplitude. Figure 1c shows results of these calculations for the $x$ and $y$ motions at different excitation frequencies. Also plotted in Figure $1 c$ is the stable stationary amplitude response to the excitation frequency calculated from eqs 33 and 34 for the same parameters. There is reasonable agreement with the trajectory calculations. The analytical calculation somewhat underestimates the amplitudes. The trajectory calculations show that there are sudden changes in the amplitudes of oscillation in both the $x$ and $y$ directions with very small changes in excitation frequency. With $x_{0}=0.1, y_{0}=0.1$, the amplitude of oscillation in $x$ decreases from a maximum at $110.934 \mathrm{kHz}$ to zero at $110.937 \mathrm{kHz}$. The y oscillation amplitude decreases from a maximum and $107.969 \mathrm{kHz}$ to zero at $107.968 \mathrm{kHz}$. In these trajectory calculations, the frequencies of the "jumps" depend on the initial conditions. When the initial positions are changed to $x_{0}=0.3$ and $y_{0}=0.3$, the "jumps" in oscillation amplitude change to 106.883 and $111.593 \mathrm{kHz}$ for the $\mathrm{y}$ and $x$ motions, respectively. The frequencies of the jumps also change with the initial $x$ and $y$ velocities (data not shown). These sudden large changes in oscillation amplitude with small changes in frequency, manifestations of bistable behavior, motivated us to investigate experimentally quadrupole excitation with linear quadrupole traps that have added octopole fields of $2.0 \%, 2.6 \%$, and $4.0 \%$.

\section{Experimental Methods}

A linear trap time-of-flight (TOF) system described in detail previously [2a] was used. Ions formed by electrospray pass through a dry nitrogen curtain gas, a 0.25 $\mathrm{mm}$ diameter orifice, into a region with a background pressure of 1.0 Torr, and then through a skimmer with a $0.75 \mathrm{~mm}$ diameter orifice. Ions then pass through a quadrupole ion guide Q0, (length $30 \mathrm{~cm}$, field radius 4.5 $\mathrm{mm}$ ) pumped to a pressure of about $3.5 \mathrm{mTorr}$, pass through an "entrance" lens with a $2.0 \mathrm{~mm}$ diameter aperture, and enter a linear quadrupole ion trap, Q1 (operating frequency $768 \mathrm{kHz}$ ) pumped to a base pressure of 3.3. $\times 10^{-6}$ Torr. The pressure in the trap can be increased above the base pressure by adding nitrogen (99.999\% stated purity, Praxair Canada Inc.) to the chamber through a needle valve. The trap pressure was measured with an ion gauge for pressures below $1 \times$ $10^{-4}$ Torr and a 0.1 Torr capacitance manometer for pressures above $1 \times 10^{-4}$ Torr. The quadrupole rod sets, Q1, have nominal added octopole fields of 2.0, 2.6, and $4.0 \%$ (Table 1 ).

Positive ions of reserpine, $m / z$ 609, are injected into the linear trap (30-100 ms fill time), confined in Q1 by applying trapping potentials to the entrance and exit apertures, cooled for $20 \mathrm{~ms}$, and are then excited for 100 $\mathrm{ms}$ at a given frequency. Ions are then cooled for $20 \mathrm{~ms}$ before mass analysis and detection in the TOF system. Finally, Q0 and Q1 are drained for $50 \mathrm{~ms}$. Table 3 shows
Table 3. Operating voltages

\begin{tabular}{ll}
\hline Sprayer & $4000 \mathrm{~V}$ \\
Curtain plate & $1.2 \mathrm{kV}$ \\
Orifice & $180 \mathrm{~V}$ \\
Skimmer & $25 \mathrm{~V}$ \\
Q0 rod offset & $15 \mathrm{~V}$ \\
Entrance lens & $+5 \mathrm{~V}$ or $+30 \mathrm{~V}$ \\
Q1 trap rod offset & $0 \mathrm{~V}$ \\
Exit lens & $-7 \mathrm{~V}$ or $+43 \mathrm{~V}$ \\
\hline
\end{tabular}

the voltages applied to various elements. To inject ions into Q1, the entrance lens was set to +1 to $+5 \mathrm{~V}$ (dependent on the pressure in $Q_{1}$ ) and to trap ions, to $+30 \mathrm{~V}$. The exit lens was set to $+43 \mathrm{~V}$ to trap ions and to $-7 \mathrm{~V}$ to allow ions to drain into the TOF analyzer.

For a given excitation amplitude, reserpine ions are excited at a given frequency. At each frequency, spectra from 100 cycles of filling the trap, exciting ions, and mass analysis were summed to determine the intensities of precursor and fragment ions. The quadrupole excitation voltage was generated by an arbitrary waveform generator (Agilent 33120m; Agilent Santa Clara, CA) and applied to the rods with a circuit described previously [4b]. All excitation voltages are reported as volts zero to peak $\left(\mathrm{V}_{0-\mathrm{p}}\right)$ pole to ground. The excitation circuit has capacitors between the quadrupole power supply and rod set. These form a capacitive voltage divider, so the rf voltage applied to the rods is less than the voltage set on the power supply. The $q$ values of the trapped ions were calculated from the resonant frequencies of the ions. The trapping $q$ of reserpine ions was chosen to be ca. 0.20 to confine both reserpine and fragment ions. All experiments were done with no dc applied between the rod pairs so the Mathieu parameter " $a$ " was zero.

Precursor ions were not isolated. At resonance, reserpine ions are depleted by fragmentation (see below). Plots of the intensity of reserpine ions and fragment ions versus excitation frequency are used to investigate the ion motion. The intensities are normalized to 1.0 for the maximum sum of all ions (precursor and fragments). This method does not directly probe the stationary amplitude response to the excitation frequency, as fragmentation is not related to oscillation amplitude in any simple way [2a, 16]. Nonetheless it gives insight into the ion motion.

Reserpine (Sigma, St. Louis, MO) was $50 \mu \mathrm{M}$ in $\mathrm{MeOH}$ (HPLC Grade; Fisher Scientific, Nepean, ON, Canada): $\mathrm{H}_{2} \mathrm{O}$ (deionized) 50:50 mixture with $0.02 \%$ acetic acid and was infused into the source at $1 \mu \mathrm{L} \mathrm{min}{ }^{-1}$.

\section{Results and Discussion}

Figure 2 shows the intensities of reserpine ions and the sum of fragment ions versus quadrupole excitation frequency for the rod sets with 2.0\% (Figure $2 \mathrm{a}$ and $\mathrm{b}$ ), $2.6 \%$ (Figure $2 \mathrm{c}$ and $\mathrm{d}$ ), and $4.0 \%$ (Figure $2 \mathrm{e}$ and $\mathrm{f}$ ), added octopole fields excited at the $K=1$ and $K=2$ resonances. (In some experiments, resonances with 


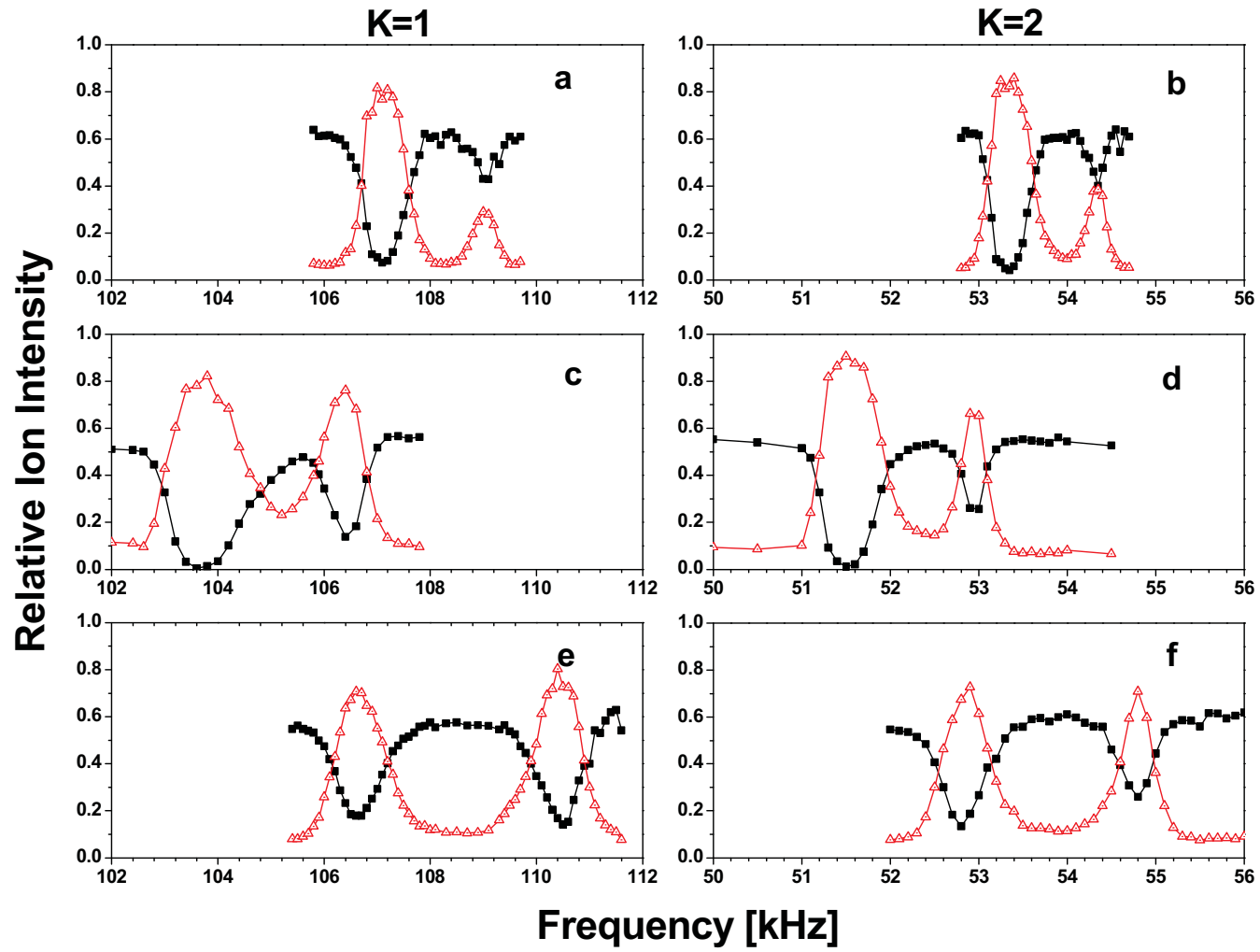

Figure 2. Ion intensities versus excitation frequency at $P=0.2$ mTorr, $q \approx 0.20$. (a) $A_{4}=$ $0.020, K=1, V_{e x}=0.20 \mathrm{~V}$; (b) $A_{4}=0.020, K=2, V_{e x}=2.0 \mathrm{~V}$; (c) $A_{4}=0.026, K=1, V_{e x}=0.30 \mathrm{~V}$; (d) $A_{4}=0.026, K=2, V_{e x}=2.25 \mathrm{~V}$; (e) $A_{4}=0.040, K=1, V_{e x}=0.40 \mathrm{~V} ;(\mathbf{f}) A_{4}=0.040, K=2, V_{e x}=$ $2.75 \mathrm{~V}$. Filled square: $m / z 609$ intensity, open triangle: sum of all fragment ions.

higher values of $K$ were investigated. However no new phenomena were found.) The trap pressure was 0.20 mTorr. The excitation voltages were chosen to give approximately equal depletions of reserpine ions in each case. The most abundant fragment ions had $\mathrm{m} / \mathrm{z}$ values of $581,448,438,397,365$, and 195, with abundances that changed with operating conditions. The maximum intensities of the sum of fragment ions exceed the initial reserpine ion intensities most likely because of mass discrimination effects in transferring the ions into and through the TOF analyzer. The $K=1$ resonances are at twice the frequency of the $K=2$ resonances, and higher voltages are required to excite the $K=2$ resonances, as expected [4b]. Our principal observation is that there are two resonances for depletion of reserpine ions, each accompanied by the forma- tion of fragment ions. In general the two resonances do not have the same strengths. The frequency differences between the two resonances increase with increasing amplitude of the octopole field.

Assuming the maximum ion depletion occurs at frequencies corresponding to the maximum stationary amplitudes, $a_{\mathrm{m}}$, can be estimated through eq 37 for the measured frequency difference $\Delta f_{m}$. These values are listed in Table 4 . In all cases $a_{m} \approx 2.3 \mathrm{~mm}$, significantly less than $r_{0}$. This, along with the observation of abundant fragment ions indicates the reserpine ions are removed mostly by dissociation and not by ejection.

With ions trapped at $q=0.20$, the fundamental frequency of oscillation is $f_{0}=54.695 \mathrm{kHz}$. Thus, the $K=2$ and $K=1$ resonances are expected to occur near 54.695 and $109.39 \mathrm{kHz}$, respectively. These frequencies

Table 4. Comparison of calculated $f_{R}-f_{L}$ with the measured difference in frequency $\Delta f$ of the two resonances for linear quadrupole ion traps with $2.0 \%, 2.6 \%$, and $4.0 \%$ added octopole fields for the $K=1$ resonances, and the amplitude, $a_{m}$, calculated from eq 37 , corresponding to the measured $\Delta f_{m}$

\begin{tabular}{|c|c|c|c|c|c|}
\hline Quadrupole & Pressure $P$ (mTorr) & Excitation $V_{e x}(\mathrm{~V})$ & Calculated $f_{R}-f_{L}(\mathrm{kHz})$ & Measured $\Delta f_{i n}(\mathrm{kHz})$ & $a_{m}$ from $\Delta f_{m}(\mathrm{~mm})$ \\
\hline \multirow[t]{2}{*}{ 2.0\% Octopole } & 0.2 & 0.20 & 1.436 & 1.95 & 2.31 \\
\hline & 1.0 & 0.25 & 1.598 & 1.90 & 2.35 \\
\hline \multirow[t]{2}{*}{$2.6 \%$ Octopole } & 0.2 & 0.30 & 2.163 & 2.55 & 2.37 \\
\hline & 1.0 & 0.35 & 2.386 & 2.50 & 2.41 \\
\hline \multirow[t]{2}{*}{ 4.0\% Octopole } & 0.2 & 0.40 & 2.887 & 3.75 & 2.27 \\
\hline & 1.0 & 0.40 & 2.766 & 4.00 & 2.30 \\
\hline
\end{tabular}




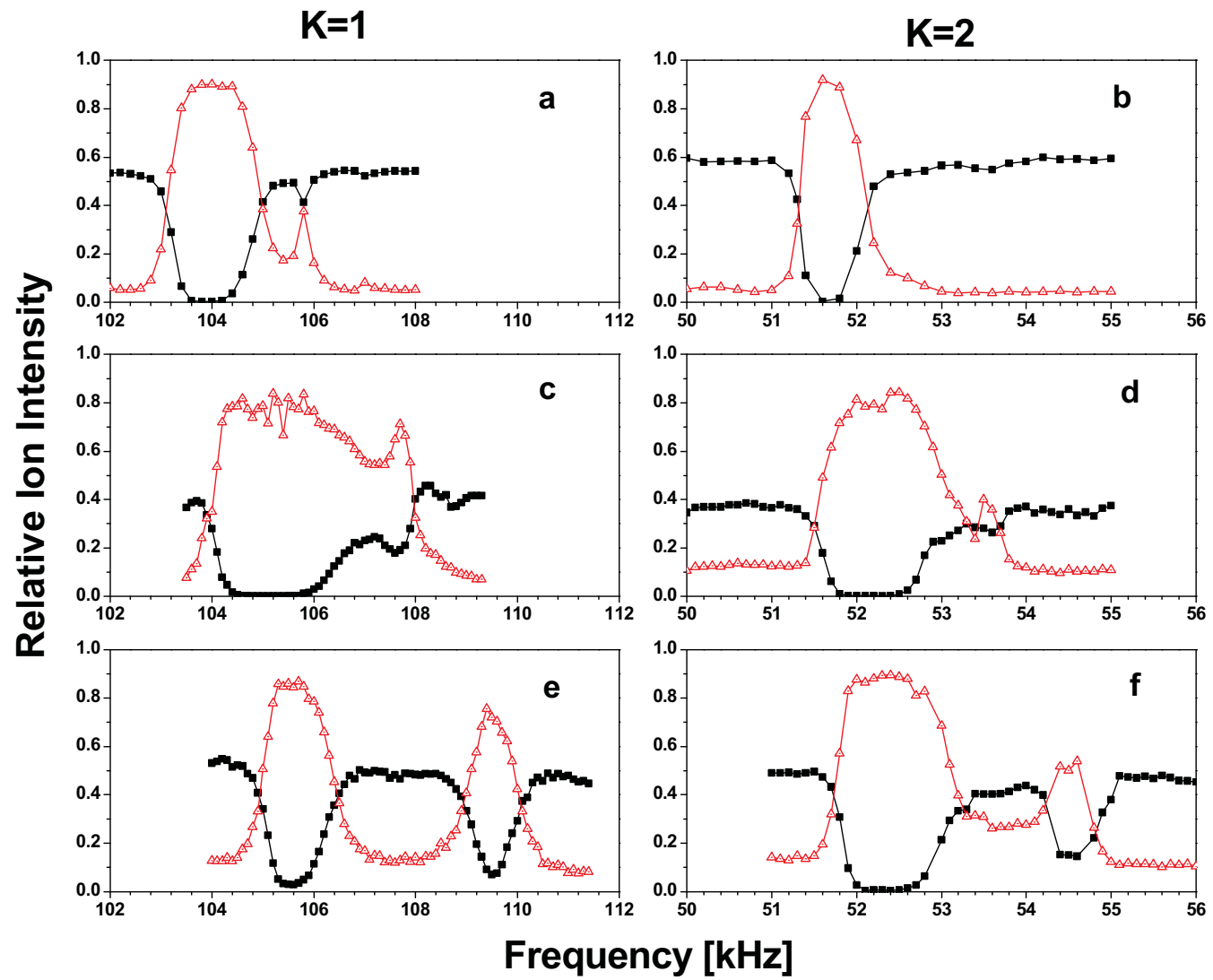

Figure 3. Ion intensities versus excitation frequency at $P=1.0 \mathrm{mTorr}, q \approx 0.20$. (a) $A_{4}=$ $0.020, K=1, V_{e x}=0.25 V$; (b) $A_{4}=0.020, K=2, V_{e x}=2.0 V$; (c) $A_{4}=0.026, K=1, V_{e x}=0.35 V$; (d) $A_{4}=0.026, K=2, V_{e x}=2.25 \mathrm{~V}$; (e) $A_{4}=0.040, K=1, V_{e x}=0.40 \mathrm{~V}$; (f) $A_{4}=0.040, K=2, V_{e x}=$ $2.85 \mathrm{~V}$. Filled square: $m / z 609$ intensity, open triangle: sum of fragment ions.

might be expected to be the center frequencies between the two resonances. In fact the center frequencies are a few percent less, indicating that the $q$ value is slightly less than 0.20 (typically 0.195), and changes slightly (ca. $\pm 1 \%$ ) between experiments done on different days, limited by the reproducibility of the electronics.

The trajectory calculations show that in some cases the maximum oscillation amplitudes occur near $f_{R}$ and $f_{L}$. Table 4 lists the measured differences in frequency between the two $K=1$ resonances and the calculated $f_{R}$ $-f_{L}$. The measured frequency differences $\left(\Delta f_{m}\right)$ are somewhat greater than $f_{R}-f_{L}$. We assign the low and high-frequency resonances to excitation of motion in the $y$ and $x$ directions respectively. Equation 37 shows the frequency difference is determined by two terms. When the amplitude of oscillation is zero, the frequency difference is $2 \sqrt{\gamma^{2} / 4-\lambda^{2}}$, and is determined by the excitation strength and damping, but not the magnitude of the octopole field. When the amplitude of oscillation increases, the frequency difference between $x$ and $y$ initially decreases, but then increases. This can be seen in Figure 1a where the curves for $a_{+}^{y}$ and $a^{x}$ first approach each other, cross, and then separate. The measured frequency differences are greater than $f_{R}-f_{L}$, indicating the ions have somewhat greater amplitudes of oscillation than those corresponding to $f_{R}$ and $f_{L}$.
At different pressures and with different operating conditions, other behavior can be seen. Figure 3 shows ion intensities versus excitation amplitude when the trap is operated at a higher pressure, $1.0 \mathrm{mTorr}\left(\mathrm{N}_{2}\right)$. The $K=1$ and $K=2$ resonances are shown for rod sets with $2.0 \%, 2.6 \%$, and $4.0 \%$ added octopole fields. In most cases, two resonances are seen. The frequency differences between the two peaks are very similar to those at 0.2 mTorr, implying the oscillation amplitudes leading to depletion and fragmentation at these two pressures are similar.

From Figure 3, clearly the low-frequency resonance can be substantially greater in intensity than the highfrequency resonance. This can also be seen somewhat in Figure 2. In some cases, e.g., Figure $3 b$, the higher frequency resonance is not seen. Simple arguments based on the potential given in eq 1 when retaining the amplitudes $A_{2}$ and $A_{4}$ only, suggest the high-frequency resonance, corresponding to the $\mathrm{x}$ motion, should have the greater depletion. First, the electric field in the $x$ direction is greater than in the y direction. Second, the effective potential well depth is greater in the $x$ direction than in the y direction. Collings [17] has shown that at $q \approx 0.2$, small increases in well depth can give relatively large increases in fragmentation efficiency (at least at ca. $3 \times 10^{-5}$ Torr). However our 

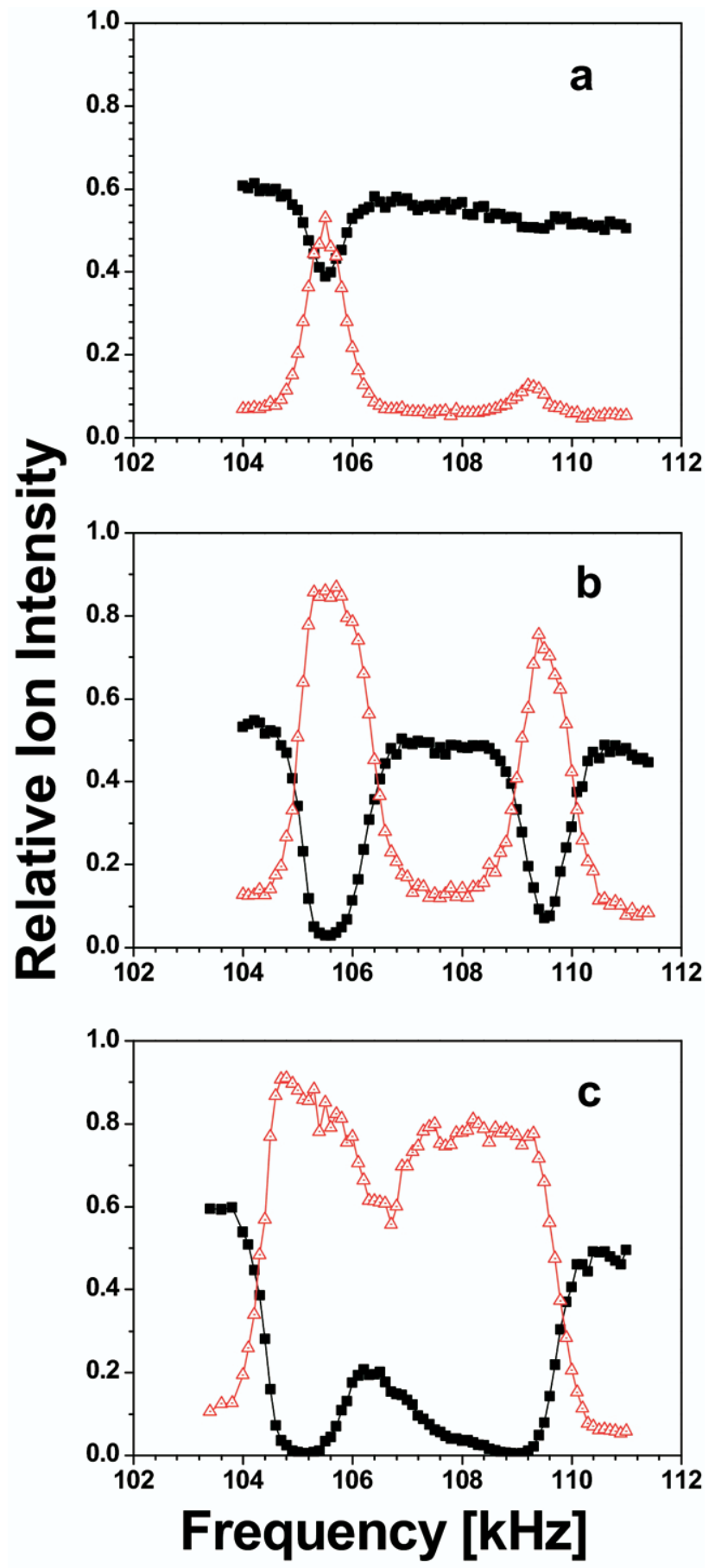

Figure 4. Ion intensities versus excitation frequency with a quadrupole with $4.0 \%$ added octopole field, $P=1.0$ mTorr, $q \approx$ $0.2, K=1$, excitation amplitudes of (a) $0.30 \mathrm{~V}$, (b) $0.40 \mathrm{~V}$, (c) $0.50 \mathrm{~V}$.

observations cannot be explained by either of these effects.

The excitation amplitude partially controls whether two resonances are seen. Figure 4 shows the effect of changing the excitation amplitude on the $K=1$ resonances at 1.0 mTorr. With $0.30 \mathrm{~V}$ amplitude, only the low-frequency resonance at $105.5 \mathrm{kHz}$ is significant, although a trace of the higher frequency resonance can be seen in the fragment yield. At $0.40 \mathrm{~V}$ the higher frequency resonance has increased in intensity and both peaks can be seen. At $0.5 \mathrm{~V}$ both peaks have grown in intensity such that the region between the peaks is filled in, and two resonances can just be seen. This is simply a saturation effect. Trajectory calculations show that under these conditions the ion oscillation amplitudes remain significantly less than $r_{0}$. A broader range of excitation frequencies give sufficient ion fragmentation to cause depletion of reserpine ions. Similar effects are seen with the rod sets with $2.0 \%$ and $2.6 \%$ added octopole fields. At higher amplitudes (data not shown) the region between the peaks fills in nearly completely and only one broad resonance is seen. Observation of the two resonances then requires a judicious choice of operating conditions.

As described, our initial motivation was to find depletion peaks that are sharp on the low-frequency side and sharp on the high-frequency side as suggested by Figure 1. We found neither. The peaks in Figures 2, 3 , and 4 are fairly symmetric, although in some case some small asymmetry is seen. With dipole excitation between the large rods of a quadrupole with $4 \%$ octopole field, an asymmetric peak sharper on the lowfrequency side is seen [2a]. However with excitation between the smaller, $x$, rods, no asymmetry is seen. The frequencies at which jumps occur will depend on the initial conditions of the ions. Because the ensemble of ions has a distribution of initial positions and velocities, our experiments may contain an average over jumps at many different frequencies. Chu et al. [7] saw sharp changes in oscillation amplitude with small changes of frequency for the collective motion of the entire ion cloud in a 3D trap. However under the same conditions, the resonance for the incoherent motion of the individual ions showed only a smooth broadened resonance with no jumps and little asymmetry. Possibly, if the ions are prepared with different initial conditions, with methods such as laser cooling [18], the resonances might show asymmetries with sharp sides. From a practical point of view, quadrupole excitation of ions in these rod sets does not offer a method of producing high-resolution ion isolation, at least with the operating conditions described here.

\section{Acknowledgments}

The authors acknowledge support for this research by the Natural Sciences and Engineering Research Council of Canada and MDS SCIEX through an Industrial Research Chair. The trajectory calculations used a program written by Mikhail (Michael) Yu. Sudakov.

\section{References}

1. Douglas, D. J.; Frank, A. J.; Mao, D. Linear Ion Traps in Mass Spectrometry. Mass Spectrom. Rev. 2005, 24, 1-29.

2. (a) Michaud, A. L.; Frank, A. J.; Ding, C.; Zhao, X.; Douglas, D. J. Ion Excitation in a Linear Quadrupole Ion Trap with an Added Octopole Field. J. Am. Soc. Mass Spectrom. 2005, 16, 835-849. (b) Collings, B. A.; Stott, W. R.; Londry, F. A. Resonant Excitation in a Low Pressure Linear 
Ion Trap. J. Am. Soc. Mass. Spectrom. 2003, 14, 622-634. (c) Collings, B. A. Increased Fragmentation Efficiency of Ions in a Low Pressure Linear Ion Trap with an Added dc Octopole Field. J. Am. Soc. Mass Spectrom. 2005, $16,1342-1352$

3. Konenkov, N.; Londry, F.; Ding, C.; Douglas, D. J. Linear Quadrupoles with Added Hexapole Fields J. Am. Soc. Mass Spectrom. 2006, 17, 1063-1073.

4. (a) Sudakov, M.; Konenkov, N.; Douglas, D. J.; Glebova, T. Excitation Frequencies of Ions Confined in a Quadrupole Field with Quadrupole Excitation. I. Am. Soc. Mass Spectrom. 2000, 11, 10-18. (b) Collings, B. A.; Douglas, D. J. Observation of Higher Order Quadrupole Excitation Frequencies in a Linear Ion Trap. J. Am. Soc. Mass Spectrom. 2000, 11, 1016-1022.

5. Alheit, R.; Chu, X. Z.; Hoefer, M.; Holzki, M.; Werth, G. Nonlinear Collective Oscillations of an Ion Cloud in a Paul Trap. Phys. Rev. A 1997, 56, 4023-4031

6. Razvi, M. A. N.; Chu, X. Z.; Alheit, R.; Werth, G.; Blumel, R. Fractional Frequency Collective Parametric Resonances of an Ion Cloud in a Paul Trap. Phys. Rev. A 1998, 58, R34-R37.

7. Chu, X. Z.; Holzki, M.; Alheit, R.; Werth, G. Observation of High-Order Motional Resonances of an Ion Cloud in a Paul trap. Int. J. Mass Spectrom. Ion Processes 1998, 173, 107-112.

8. Tommaseo, G.; Paasche, P.; Angelescu, C.; Werth, G. Subharmonic Excitation of the Eigenmodes of Charged Particles in a Penning Trap. Eur. Phys. J. D 2004, 28, 39-48.

9. Paasche, P.; Valenzuela, T.; Biswas, D.; Agelescu, C.; Werth, G. Individual and Center-of-Mass Resonances in the Motional Spectrum of an Electron Cloud in a Penning Trap. Eur. Phys. J. D 2002, 18, 295-300.
10. Ding, L.; Sudakov, M.; Brancia F. L.; Giles, R.; Kumashiro, S. A Digital Ion Trap Mass Spectrometer Coupled with Atmospheric Pressure Ion Sources. J. Mass Spectrom. 2004, 39, 471-484.

11. (a) Sudakov, M. Yu.; Douglas, D. J. Linear Quadrupoles with Added Octopole Fields. Rapid Commun. Mass Spectrom. 2003, 17, 2290-2294. (b)Ding, C.; Konenkov, N. V.; Douglas, D. J. Quadrupole Mass Filters with Octopole Fields. Rapid Commun. Mass Spectrom. 2003, 17, 24952502.

12. (a) Dehmelt, H. G. Radiofrequency Spectroscopy of Stored Ions. I: Storage Adv. Atom. Mol. Phys. 1967 3, 53. (b) Gerlich, D. Inhomogeneous RF Fields: A Versatile Tool for the Study of Processes with Slow Ion. In Advances in Chemical Physics, Vol. LXXXII; John Wiley and Sons: New York, 1992; pp 1-176.

13. Shivamoggi, Bhimsen K. Perturbation Methods for Differential Equations; Birkauser: Boston, 2002; Chap VI, p 219.

14. Thomsen J. J. Vibrations and Stability, 2nd ed.; Springer Verlag: Berlin, 2003; p 105.

15. Landau, L. D.; Lifshitz, E. M. 1960 Mechanics, 3rd ed.; Pergamon Press: New York, 1960; pp 74-93.

16. (a) Campbell, J. M.; Collings, B. A.; Douglas, D. J. A Linear Ion Trap Time-of-Flight System with Tandem Mass Spectrometry Capabilities. Rapid Commun. Mass Spectrom. 1998, 12, 1463-1474. (b) Collings, B. A. Campbell, J. M.; Mao, D.; Douglas, D. J. A Combined Linear Ion Trap Time-of-Flight System with Improved Performance and MS $^{n}$ Capabilities. Rapid Commun. Mass Spectrom. 2001, 15, 1777-1795.

17. Collings, B. A. Fragmentation of Ions in a Low Pressure Linear Ion Trap. J. Am. Soc. Mass Spectrom. 2007, 18, 1459-1466.

18. Drakoudis, A.; Sollner, M.; Werth, G. Instabilities of Ion Motion in a Linear Paul Trap. Int. J. Mass Spectrom. 2006, 252, 61-68. 